\title{
AVALIAÇÃO ANTROPOMÉTRICA DE PACIENTES INTERNADOS EM UMA UNIDADE DE TERAPIA INTENSIVA EM CASCAVEL/PR
}

\section{ANTHROPOMETRIC EVALUATION OF INJURED PATIENTS IN A UNIT OF INTENSIVE THERAPY IN CASCAVEL / PR}

\author{
Larissa Cristina da Silva ${ }^{1}$, Débora Regina Hendges Poletto Pappen ${ }^{2}$ \\ ${ }^{1}$ Nutricionista, Centro Universitário Fundação Assis Gurgacz (FAG). ${ }^{2}$ Mestre. Docente do Curso de \\ Nutrição, Centro Universitário Fundação Assis Gurgacz (FAG). \\ * Autor correspondente: de poletto@hotmail.com, https://orcid.org/0000-0003-0643-0346
}

\begin{abstract}
RESUMO
Unidade de Terapia Intensiva encontrasse diversos tipos de pacientes com quadros nutricionais diversos, e como respondem a tipo de tratamento. Destacando-se sobre suas modificações metabólicas, o papel que a nutrição exerce com a avaliação antropométrica. Diante dessa Importância o objetivo deste trabalho foi avaliar o estado nutricional, através de medidas antropométricas, para que haja melhorias na conduta dietoterápica, para a fim de melhorar ou recuperar o quadro nutricional do paciente. Esse estudo e feito através da coleta de dados que se baseou no sexo, idade, diagnóstico, peso $(\mathrm{kg})$, estatura $(\mathrm{m})$, IMC, CB $(\mathrm{cm})$, CMB, PCT $(\mathrm{mm})$, e IMC. Após tabular os dados foi feita a classificado do estado nutricional dos indivíduos. Dentre todos os 14 pacientes observou-se predominância do gênero masculino, com idades entre 32 a 77 anos, com diagnóstico nutricional de eutrofia através do IMC (Índice de massa corporal) em adultos e idosos e a partir da adequação da prega cutânea tricipital os pacientes apresentaram $8 \%$ de obesidade e $3 \%$ de desnutrição grave. Já a adequação de circunferência de braço apresentou 4\% dos pacientes em eutrofia e 4\% com desnutrição moderada. Diante disso, é possível observar a importância da avaliação antropométrica que resultará em benefícios para esses pacientes através do diagnósticos. Palavras-chave: Avaliação Antropométrica, Pacientes Críticos,
\end{abstract} Unidade de Terapia Intensiva.

\section{ABSTRACT}

The intensive care unit involves several types of patients with the disease, and as a type of treatment. Highlighting their metabolic role, nutrition plays an anthropometric role. Diverge the Importance of the objective of this nutritional status study, through anthropometric measures, so that the diet is predicted, in order to improve or recover the patient's nutritional status. (B), BMI (BMI), BMI (BMI), BMI (BMI), WBC (BMI), PCT $(\mathrm{mm})$, and BMI. After tabulation the data were made for nutritional classification of the individuals. Among all 14 patients, the predominance of males, aged between 32 and 77 years, with nutritional diagnosis of eutrophy through BMI in adults and the elderly, was observed, and from the adaptation of the tricipital skinfold fold $8 \%$ of obesity and $3 \%$ severe malnutrition. The adequacy of the circumference of $4 \%$ of patients in eutrophy and $4 \%$ with moderate malnutrition was done. Given this, it is possible to observe the anthropometric evaluation that results in benefits for the patients through diagnoses. Keywords: Anthropometric Evaluation, Critical Patients, Intensive Care Unit. 


\section{INTRODUÇÃO}

Pacientes em estado crítico apresentam maior incidência a riscos, havendo perdas e falência de várias partes dos sistemas que compõem o corpo humano. Esses indivíduos tendem a piorar na fase aguda da patologia acometida, ocorrendo um aumento na degradação de micronutrientes e macronutrientes, os quais são essenciais para homeostasia normal do organismo. Algumas complicações podem surgir como a resistência à insulina e uma possível sobrecarga do sistema linfático (LAMEU, 2005; KREYMANN, 2006).

Há uma incidência no aumento de 20 a 50\% de desnutrição em pacientes hospitalizados, havendo uma piora do quadro dessas patologias (CORREIA, CAMPOS, 2003; NORMAN, 2008; RASMUSSEN, 2010).

Através destas informações a desnutrição e ocasionado por diminuição de ingesta alimentar ou pela mesma não suprir demanda nutricional do paciente, pela dificuldade dos mesmos na deglutição e mastigação dos alimentos ofertados e a diminuição da absorção de micro e macronutrientes devido à interação medicamentosa, esses fatores podem atrapalhar assim na recuperação e melhora do quadro nutricional dos pacientes (MAICÁ, 2008).

Há uma grande quantidade de pacientes internados na UTI que estão confinados ao leito, sem possibilidade de movimentação, debilitando assim cada fez mais a musculatura, podendo levar a um quadro de imobilidade após a alta (NANAS, 2008). Essas alterações podem ocasionar inflamação sistêmica no organismo junto com modificações metabólicas dos componentes das vias energéticas, que geram uma grande perda da massa muscular magra, devido ao stress do metabolismo no estado anabólico que acontece para suprir a demanda nutricional. Esse efeito é intitulado alostático de estresse, que acaba colaborando para que o corpo entre em falência devido à obstrução de diversos órgãos (PLANK, 2003).

A Avaliação nutricional em adultos em geral é feita através da verificação da composição corporal, a mesma necessita de técnicas para análise das reservas energéticas, massa muscular e metabólica. São diversos os processos para definir o componente do peso corporal, entre elas possui as medidas antropométricas, sua utilização e muito frequente. Para coletar os dados, possui baixo custo e são simples de avaliar (ANJOS, 1992; MONTEIRO, 1998; WORLD, 1998).

Para avaliar a quantidade de estoque proteica e energética do indivíduo, utilizasse CB (circunferência do braço), CMB (circunferência muscular do braço), CP (circunferência da panturrilha) e PCT (prega cutânea tricipital). A Prega Cutânea Tricipital mostra a quantidade de tecido adiposo como a circunferência do braço, a prega junto com a circunferência se obtém circunferência muscular do braço para o cálculo de massa muscular. Há também outros parâmetros para análise, o tipo dependera da necessidade de cada paciente (FONSECA, SANTOS, 2013).

O objetivo deste trabalho foi avaliar o estado nutricional, através de medidas antropométricas, para que haja melhorias na conduta dietoterápica, para a fim de favorecer ou recuperar o quadro nutricional do paciente.

\section{METODOLOGIA}

O presente estudo é uma pesquisa de campo envolvendo pacientes da UTI de um hospital do oeste do Paraná, com avaliação de ambos os sexos e acima de 20 anos de idade. $\mathrm{Na}$ amostra teve 20 pacientes, pois foram avaliados só os pacientes que se encontravam internados nos dias do procedimento, a coleta foi feita no período 
de maio até junho de 2018. Este projeto foi avaliado e aprovado pelo Comitê de Ética e Pesquisa do Centro Universitário FAG sob parecer $n . \stackrel{0}{2}$ 2.645.422.

A participação dos pacientes para a pesquisa foi instruída através da aceitação e autorização assinada pelos mesmos, no Termo de Consentimento Livre e Esclarecido TCLE. Após a explicação e o objetivo da pesquisa para os pacientes e após a assinatura, foi feita a avaliação antropométrica.

Onde através de formulários específicos, que contem data, sexo, nome do paciente (somente iniciais), data de nascimento, idade, diagnóstico, peso $(\mathrm{kg})$, estatura $(\mathrm{m})$, CB $(\mathrm{cm})$, PCT $(\mathrm{mm})$, e IMC $\left(\mathrm{Kg} / \mathrm{m}^{2}\right)$. Os pacientes foram avaliados nos próprios leitos da UTI, utilizando uma fita medrica 1,50 metros, Adipômetro Plicômetro Científico Skinfold Caliper - Sahean. Já o peso e altura foram coletados através da planilha e prontuário do paciente.

A Avaliação do estado nutricional por medidas antropométricas foram os cálculos do IMC (Índice de Massa Corporal) onde foi coletado o peso e altura dos pacientes críticos, a CB (Circunferência do Braço), CMB (Circunferência Muscular do Braço), PCT (Prega Cutânea Tricipital). Uma dobra cutânea e a circunferência verificam o estoque proteico e energético dos indivíduos (FONSECA, SANTOS, 2013). Já o Índice de Massa Corporal concede o grau de estado nutricional através OMS (1998) para adultos e idosos a tabela de Lipschitz (1994).

Quadro 1 - Fórmula para o cálculo da circunferência muscular do braço

IMC: Peso Atual (kg) / Estatura $(\mathrm{m})^{2}$

A CB (circunferência do braço) foi aferida através de uma fita de medidas antropométrica inelástica, na aferição o paciente vergou o braço, posicionando na frente do tórax onde formulou um ângulo de $90^{\circ}$, que se encontrou um ponto médio entre acrômio e o olecrano marcando o local, o indivíduo ficou com o braço esticado ao longo do corpo com a palma da mão voltada para a coxa. A fita de medidas fez 0 contorno do braço no local definido de maneira que se ajuste, assim evitando aperto da pele ou folga. Após a coleta de dados ouve a realização da adequação da CB segundo gênero e idade de acordo com o percentil 50 da tabela de Frisancho (1990).

Quadro 2- Fórmula para o cálculo da adequação da circunferência do braço

$$
\text { Adequação da CB }(\%)=\frac{\text { CB obtida }(\mathrm{cm}) \times 100}{\text { CB percentil } 50}
$$

A CMB (circunferência muscular do braço) foi realizada através do cálculo que avalia a reserva de tecido muscular com os valores obtidos da CB e a PCT. Após o mesmo houve a adequação da CMB segundo gênero e idade de acordo com o percentil 50 da tabela de Frisancho (1990) e para idosos segundo gênero e idade de acordo com o percentil 50 da Método de Burr \& Phillips (1984) e a classificação do estado nutricional conforme a tabela de Blackburn, G.L. \& Thornton,P.A.,(1979). 
Quadro 3 - Fórmula para o cálculo da circunferência muscular do braço

$$
\mathrm{CMB}(\mathrm{cm})=\mathrm{CB}(\mathrm{cm})-\pi(3,14) \times[\mathrm{PCT}(\mathrm{mm})]
$$

Quadro 4 - Fórmula para o cálculo de adequação da circunferência muscular do braço

$$
\text { Adequação da CMB }(\%)=\frac{\text { CMB obtida }(\mathrm{cm}) \times 100}{\mathrm{CMB} \text { percentil } 50}
$$

A PCT (prega cutânea tricipital) foi verificada através de Plicômetro (Adipômetro) científico, onde o mesmo vai estar retilíneo no local definido da Circunferência do Braço que foi separada parcialmente a prega do mesmo, assim soltando o tecido muscular. Após a coleta houve a adequação da PCT (Dobra cutânea tricipital) para adultos segundo gênero e idade de acordo com o percentil 50 da tabela de Frisancho (1990) e para idosos segundo gênero e idade de acordo com o percentil 50 da tabela de Burr \& Phillips (1984). Já o diagnostico de classificação do estado nutricional foi conforme a tabela de Blackburn \& Thornton (1979).

Quadro 5 - Fórmula para o cálculo de adequação prega cutânea tricipital.

$$
\text { Adequação da PCT }(\%)=\frac{\text { PCT obtida }(\mathrm{cm}) \times 100}{\text { PCT percentil } 50}
$$

Os dados coletados foram armazenados e tabulados em uma planilha no programa Microsoft Office Excel 2010.

\section{RESULTADOS E DISCUSSÕES}

No período de maio de 2018, foram avaliados 14 de pacientes na UTI, 5 mulheres e 9 homens a idade de ambos variou entre 32 e 77 anos.

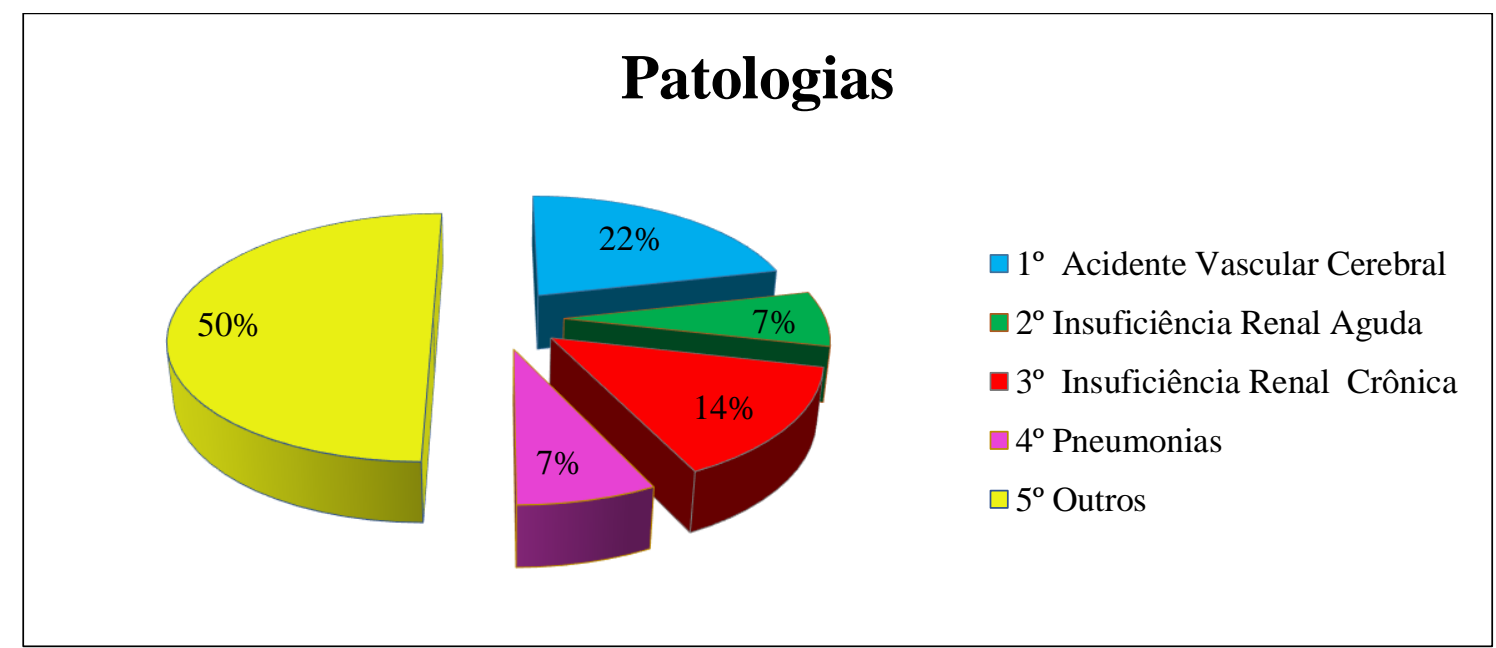

Figura 1. Distribuição da quantidade de patologias mais acometidas em uma unidade de terapia intensiva. 
Com essa amostra, observou-se que a maioria dos pacientes internados em uma unidade de terapia intensiva esta sendo constituída de $50 \%$ outras patologias, $22 \%$ Acidente vascular cerebral, 14\% Insuficiência Renal Crônica, 7\% Insuficiência Renal Aguda e 7\% Pneumonias como mostra o gráfico acima.

Segundo Martins et al.,(2017) cerca de 25\% dos pacientes apresentam outras patologias, 14,3\% acidente vascular cerebral, 7,1\% pneumonia. Já estudos de Favarin et al.,(2012) buscaram sobre as causas que levaram à internação de indivíduos em uma unidade de terapia intensiva, as principais causas foram doenças infecciosas $28 \%$ e as neurológicas em $25 \%$, doenças metabólicas $12 \%$ e por outras causas $5 \%$.

\section{IMC Adultos}

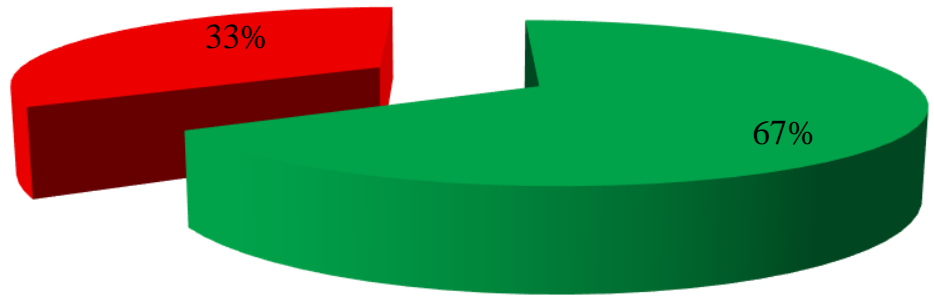

- Magreza

Eutrofia

- Sobrepeso

- Obesidade I

Obesidade II

- Obesidade III

Figura 2. Caracterização da amostra do estado nutricional através do Índice de Massa Corporal em adultos (IMC).

O Diagnóstico de eutrofia (62\%) possui um número superior aos outros resultados, trazendo o favorecimento do quadro nutricional dos pacientes, garantindo uma melhora na recuperação dos indivíduos que se encontram naquele meio. Em segundo observou-se um elevado índice de sobrepeso o qual requer atenção, pois pode ser agravado para obesidade grau III.

Segundo Martins et al.,(2017) cerca de 46\% dos pacientes internados em uma unidade de terapia intensiva apresentaram eutrofia. Porem deve-se levar em conta 0 processo inflamatório devido à depleção proteica, que aumenta assim consecutivamente o liquido extracelular gerando retenção hídrica. Observou-se resultado semelhante no estudo de Stefanello et al.,(2014) com a prevalência 44,44\% dos indivíduos em classificação de eutrofia, e em seguida com excesso de peso e por ultimo magreza, assim como o resultado de Huang et al.,(2000) seus estudos mostraram que 49 pacientes críticos avaliados apresentaram eutrofia pelo índice de Massa Corporal. Já Araujo et al.,(2011) apresentou 11 pacientes que se encontravam com sobrepeso. Segundo ele a maioria dos indivíduos estava com edema.

Os resultados mostrando nesse estudo como o sobrepeso deve ser levado também a uma aflição nutricional, pois pode haver interferência na recuperação do indivíduo assim como o desenvolvimento de outras patologias.

Já os idosos apresentaram uma classificação semelhante aos adultos, porém não houve nenhum quadro de excesso de peso, logo abaixo se encontra o gráfico 2 com os resultados. 


\section{IMC Idosos}

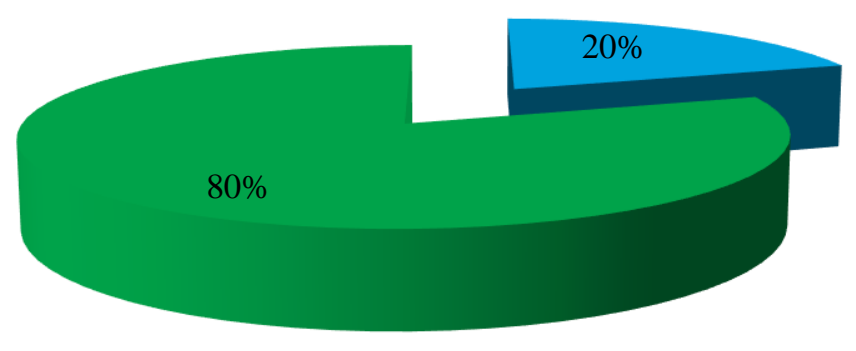

- Magreza

- Eutrofia

- Excesso de peso

Figura 3. Caracterização da amostra do estado nutricional através do Índice de Massa Corporal em idosos (IMC)

Em relação aos estudos de Santos et al.,(2005) que analisou faixas etárias de 60 a 80 anos que são as idades onde ocorrem maior alteração do estado nutricional, a maioria desses participantes foram do sexo feminino, e $60 \%$ dessas mulheres apresentaram excesso de peso. Segundo o autor a algumas controversas sobre 0 estado nutricional devido a o processo de envelhecimento estar relacionada ao acumulo de gordura subcutânea e visceral, assim havendo o aumento da massa corporal. Podendo garrar quadros diferentes no tipo físico de homens e mulheres e alguns fatores genéticos podem estar relacionados.

Já estudos de Benedetti et al.,(2012) em ralação ao índice de massa corporal apresentou que $60,4 \%$ dos idosos de ambos os sexos apresentaram excesso de peso, porem os acima de 80 anos apresentaram eutrofia.

Porem esse panorama apresenta que $80 \%$ dos idosos de ambos os sexos e idade acima de 65 anos apresentaram eutrofia, por outro lado $20 \%$ apresentaram magreza. O diagnóstico do IMC pode mascarar o verdadeiro estado nutricional, pois apresenta particularidades conforme o envelhecimento como a diminuição da altura, redução da massa magra, e redução da quantidade de agua do organismo. Essas alterações somadas com as patologias levam em discussão sobre o uso do índice de massa corporal segundo os autores citados a cima.

Para assim haver um melhor diagnostico houve a necessidade de avaliar a Prega Cutânea Tricipital, Circunferência do Braço e Circunferência Muscular do Braço que estão nos gráficos apresentados na figura 4 e 5. 


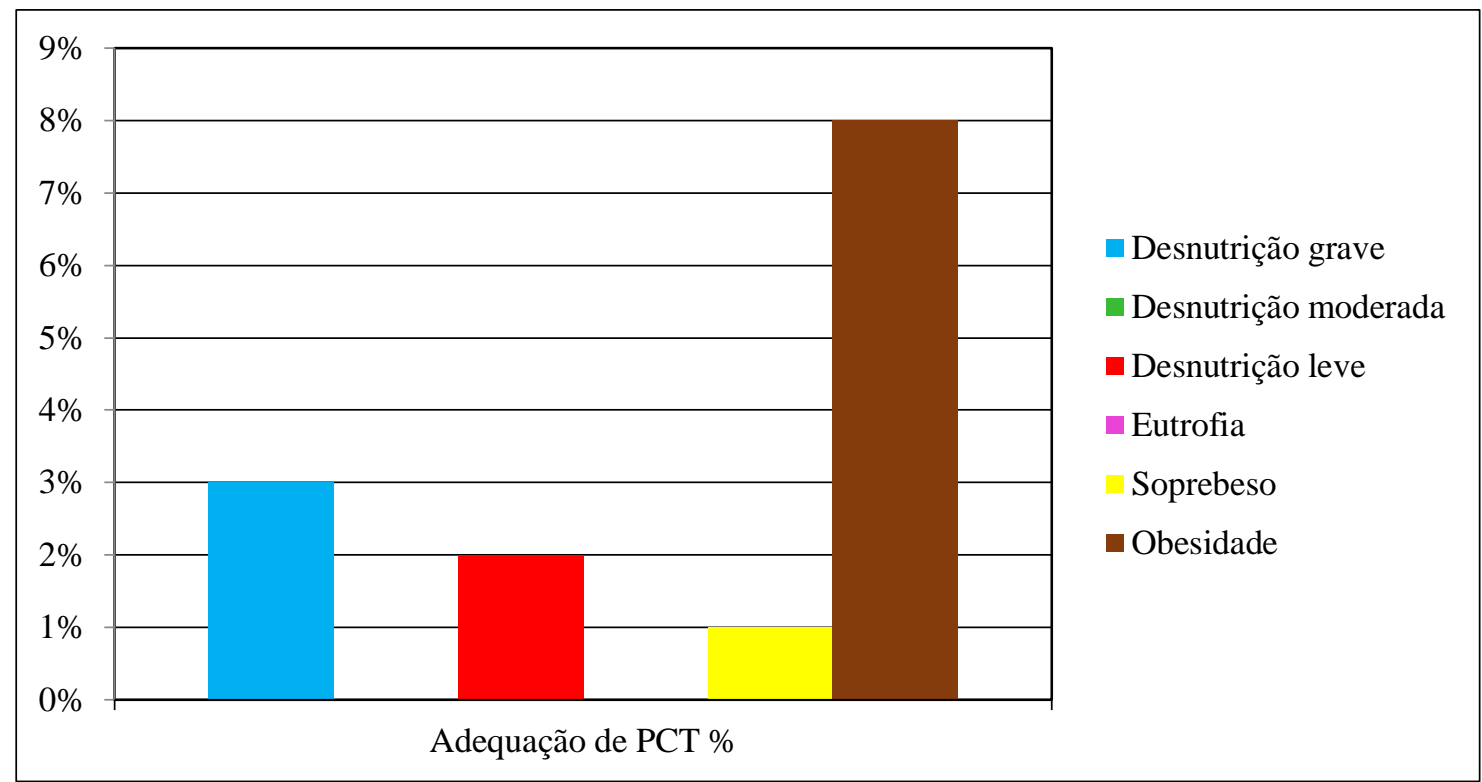

Figura 4. Classificação da amostra do estado nutricional através e adequação \% da PCT (Prega Cutânea Tricipital)

Através de estudos de Martins et al.,(2017) mais de 56,4\% dos pacientes apresentaram desnutrição e $43,45 \%$ obesidade. Através da adequação pode-se notar uma transição entre desnutrição e obesidade, essa variação se da devido a o tipo de patologia acometida pelo indivíduo principalmente em idosos. Já estudos de Souza et al.,(2009) $55 \%$ dos pacientes de ambos os sexos apresentaram um quadro de eutrofia.

A adequação da prega cutânea tricipital no gráfico mostra que $8 \%$ dos pacientes apresentam obesidade e 3\% com desnutrição grave. Resultados semelhantes a estudos citados logo acima, podendo mostrar o extremo de dois tipos de diagnostico que podem proporcionar maiores danos à saúde dos acometidos como piora na recuperação dos mesmos.

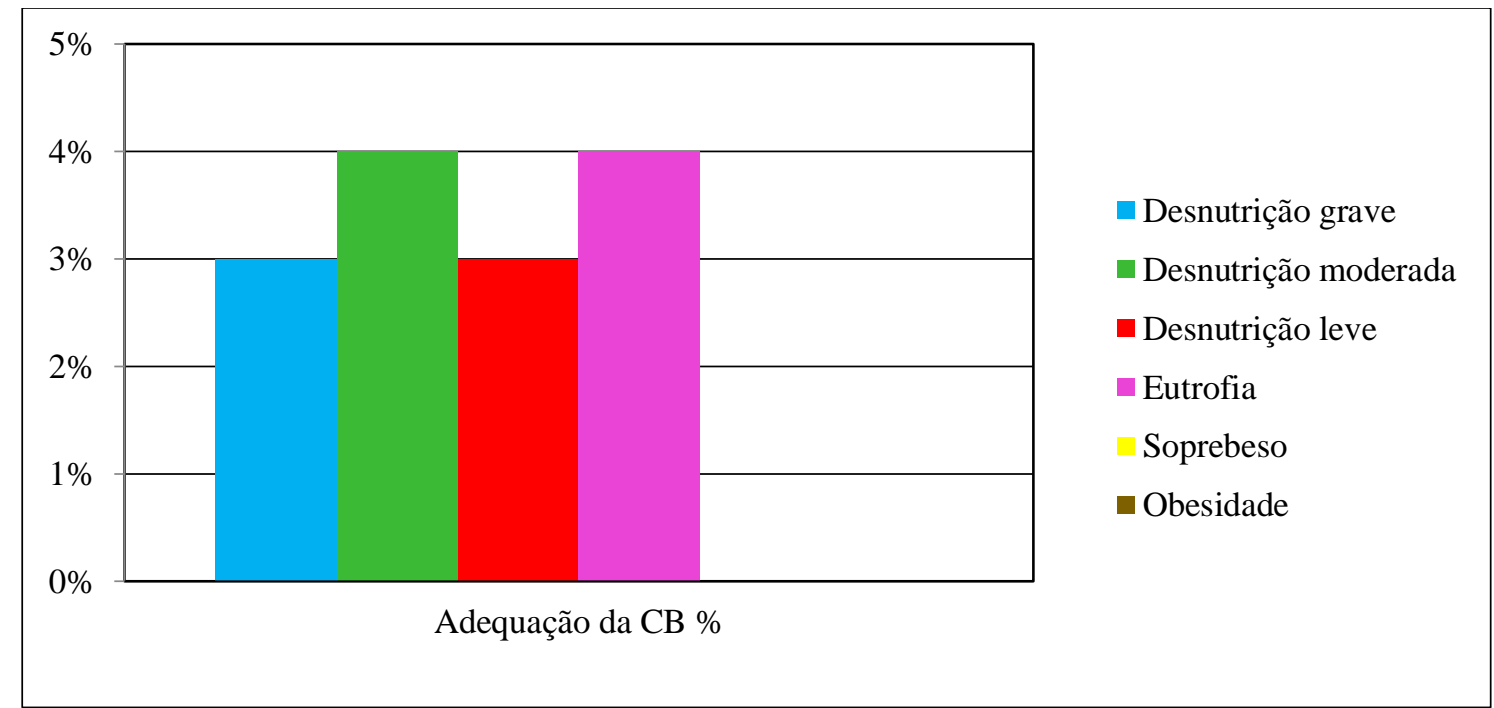

Figura 5. Classificação da amostra do estado nutricional através e adequação \% da CB (circunferência do braço) 
Conforme pesquisas de Fontora et al.,(2006) sua análise apresentou que 50\% dos pacientes avaliados apresentava desnutrição. Semelhante ao estudo de Ravasco et al.,(2002) que mostrou através da adequação CB que $43 \%$ dos indivíduos internados também estavam com desnutrição.

O gráfico apresentado na Figura 6 mostra que 4\% apresentaram eutrofia e desnutrição moderada e apenas $3 \%$ desnutrição grave e desnutrição leve.

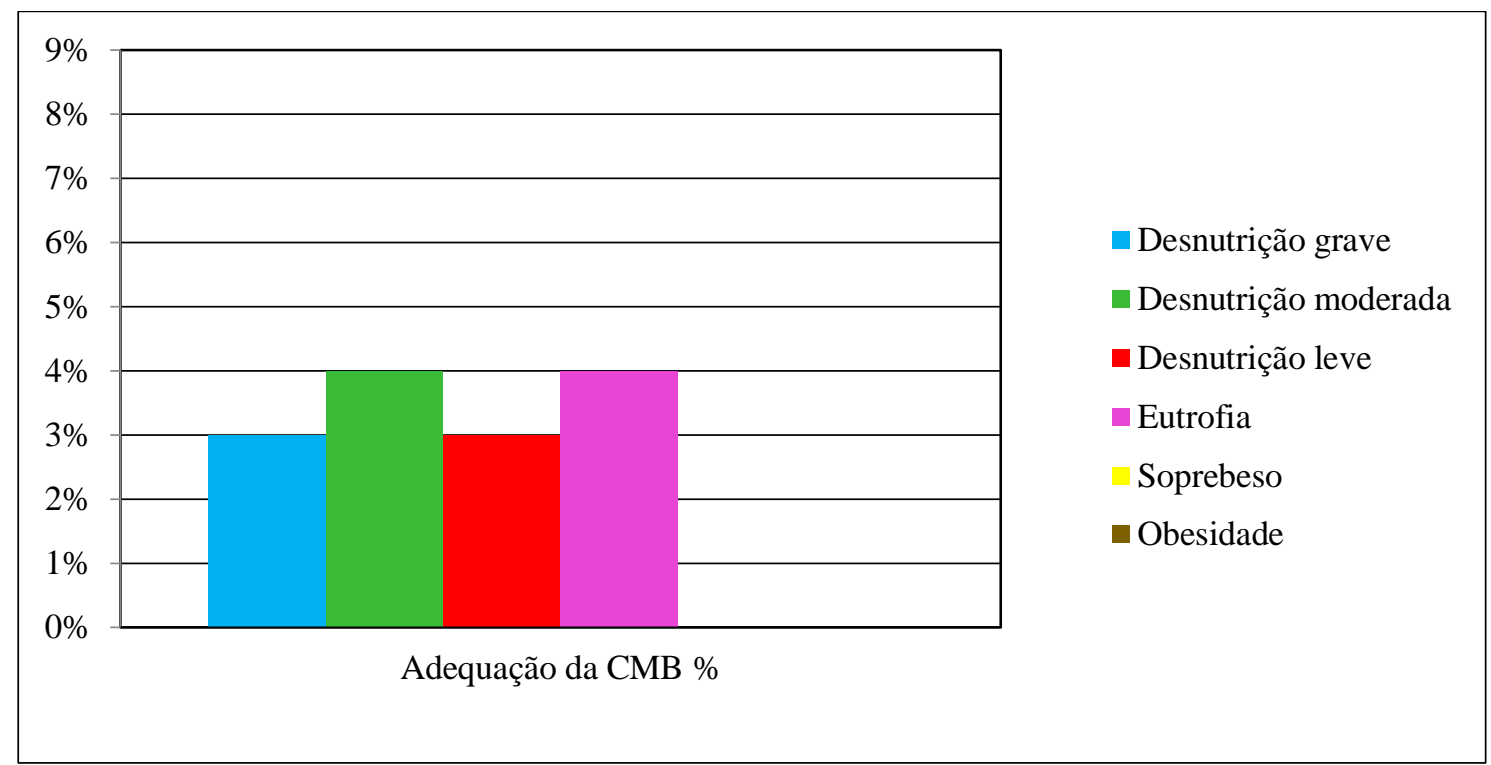

Figura 6. Classificação da amostra do estado nutricional através e adequação \% da CMB (circunferência muscular do braço)

Conforme ilustrado no gráfico 6 , foi constatado que $4 \%$ dos pacientes apresentaram eutrofia e desnutrição moderada e 3\% desnutrição grave e desnutrição leve. O diagnóstico e devido à imobilidade e incapacidade de pacientes que se encontram confinados ao leito, gerando assim um grande stress catabólico devido as patologias acometidas pelos mesmos, agravando desse modo a perda de massa muscular.

De acordo com estudos de Souza et al.,(2009) foram avaliados 55\% indivíduos sendo eles $25 \%$ com desnutrição grave e $25 \%$ com desnutrição moderada, isso pode esta relacionado com a diminuição de massa muscular magra. Resultados semelhantes foram apontados por Fontora et al.,(2006) que cerca de 55\% dos pacientes apresentaram desnutrição moderada e 38\% grave, a mesma teve maior predominância em pacientes idosos acima de 65 anos.

\section{CONCLUSÃO}

A avaliação antropométrica realizada mostrou que maioria dos pacientes críticos internados em uma unidade de terapia intensiva está com desnutrição e ao extremo como a obesidade. Podendo justificar esse quadro como o tipo de tratamento clinico e nutricional utilizado e as alterações metabólicas.

Mostrando que os pacientes críticos necessitam de uma intervenção nutricional rápida e de acompanhamento diário para melhora do estado nutricional e quadro clinico. 


\section{REFERÊNCIAS}

ARAÚJO M., dE ARAÚJO M. F. M., CAETANO O. A., GALVÃO M.T. G., DAMASCENO M. M. C. Diagnósticos de enfermagem para pacientes em risco de desenvolver úlcera por pressão. Revista Brasileira de Enfermagem, v. 64, n. 4, 2011.

ANJOS LA. Índice de massa corporal (massa corporal.estatura-2) como indicador do estado nutricional de adultos: revisão de literatura. Rev Saúde Pública 1992; 26:4316.

BLACKBURN, G. L.; THORNTON, P. A. Nutritional assessment of the hospitalized patient. The Medical Clinics of North America, v. 63, n. 5, p. 11103-11115, 1979.

BURR, M. L.; PHILLIPS, K.M. Anthropometric norms in the elderly. Br J Nutr, v. 51, n. 2, p. 165-9, 1984.

CORREIA, M. I.; CAMPOS, A. C. Prevalence of hospital malnutrition in LatinAmerica: the multicenter ELAN study. Nutrition, v. 19, n. 10, p. 823-825, oct. 2003.

CHUMLEA, W. C.; ROCHE, A. F.; STEINBAUGH, M. L. Estimating stature from knee height for persons 60 to 90 years of age. Journal of the American Geriatrics So ciety, v. 33, n. 2, p. 116-120, 1985.

FAVARIN, S. S.; CAMPONOGARA, S. Perfil dos pacientes internados na unidade de terapia intensiva adulto de um hospital universitário. Revista de Enfermagem da UFSM, v. 2, n. 2, p. 320-329, 2012.

FONSECA, J.; SANTOS, A. Anatomia aplicada à clínica: antropometria na avaliação nutricional de 367 adultos submetidos a gastrostomia endoscópica. Acta. Med. Port. 2013 Mai/Jun, 26(3): 212-18.

FONTOURA C. S. M., CRUZ D. O., LONDERO L. G., VIEIRA, R. M., Avaliação nutricional de paciente crítico. Rev Bras Ter Intensiva, v. 18, n. 3, p. 298-306, 2006.

FRISANCHO, A. R. Anthropometric Standards for the Assessment of Growth and Nutritional Status. Ann Arbor, Michigan: University of Michigan Press, 1990.

GUGELMIN S. A., SANTOS R.V. uso do índice de massa corporal na avaliação do estado nutricional de adultos indígenas xavánte, terra indígena sangradouro-Volta Grande, Mato Grosso, Brasil. Cad. Saúde Pública, Rio De Janeiro, 22(9):1865-1872, Set, 2006

HUANG Y.C., - Malnutrition in the critically ill. Nutrition, 2001;17:745-746 14. Heyward VH, Stolarczyk LM - Método de Impedância Bioelétrica Avaliação da Composição Corporal Aplicada. São Paulo: Manole, 2000.

KREYMANN KG, BERGER MM, DEUTZ NEP, HIESMAYR $M$, JOLLIET $P$, KAZANDJIEV G, ET AL. ESPEN Guidelines on enteral nutrition: intensive care. Clin Nutr. 2006;25(2):210-23. 
LAMEU E. CLÍNICA NUTRICIONAL. 1a ed. Rio de janeiro: revinter; 2005. P.787-806 LIPSCHITZ, DA. SCREENING FOR NUTRITIONAL STATUS IN THE ELDERLY. PRIM CARE 1994; 21:55- 67.

LIPSCHITZ, D.A. Screening for nutritional status in the elderly. Prim Care, 1994.

LOPES M. C. B. R. Correlação entre as atividades da equipe multiprofissional em terapia nutricional e a administração da nutrição enteral em unidades públicas de Terapia Intensiva o Distrito Federal. Repositório Institucional Da Unb 2016

MAICÁ A.O., SCHWEIGERT I.D. Avaliação nutricional em pacientes graves. Rev Bras Ter Intensiva, 2008; 20(3):286-295

MARTINS, R. C. F. Perfil nutricional de pacientes internados em unidade de terapia intensiva. Nutricion Clinica y Dietetica Hospitalaria, v. 37, p. 40-47, 2017.

MONTEIRO JC. Obesidade: diagnóstico, métodos e fundamentos. In: Halpern A, Matos AFG, Suplicy HL, Mancini MC, Zanella MT, organizadores. Obesidade. São Paulo: Lemos Editoral; 1998. p. 31-53.

NANAS S, KRITIKOS K, ANGELOPOULOS E, SIAFAKA A, TSIKRIKI S, PORIAZI M,. Fatores predisponentes para polêmutiomiopatia de doença crítica em uma unidade de terapia intensiva multidisciplinar. Acta Neurol Scand. 2008; 118 (3): 17581

NORMAN, K., OLIVEIRA, N. S. Prognostic impact of disease-related malnutrition. Clin Nutr, v. 27, n. 1, p. 5-15, feb. 2008. Enteral Nutrition Therapy in ICU: longitudinal followup. Nutrire. Rev Soc Bras Alim Nutr, v. 35, n. 3, p. 133-148, dez. 2010.

Organização Mundial de Saúde-OMS. Obesity: preventing and managing the global epidemic. Report of a WHO consultation, Geneva, 3-5 Jun 1997. Geneva: World Health Organization, 1998.

PLANK LD, HILL GL. Energy balance in critical illness. Proc Nutr Soc, 2003; 62: 545552.

QUETELET, Adolphe. Anthropometrie. 1870.

RASMUSSEN, H. et al. Measuring nutritional risk in hospitals. Clin Epidemiol, v. 2, p. 209-216, oct. 2010.

RAVASCO P, CAMILO M.E., GOUVEIA-OLIVEIRA A ., - A critical approach to nutritional assessment in critically ill patients. Clin Nutr, 2002;21:73-77.

SANTOS, D. M. D.; SICHIERI, R.. Índice de massa corporal e indicadores antropométricos de adiposidade em idosos. Revista de saude pública, v. 39, p. 163168, 2005.

SOUZA, S. M. P.. Nutritional profile of lung transplant candidates. Jornal Brasileiro de Pneumologia, v. 35, n. 3, p. 242-247, 2009. 
STEFANELLO M.D., POLL F.A., Estado nutricional e dieta enteral prescrita e recebida por pacientes de uma Unidade de Terapia Intensiva. ABCS Health Sciences, v. 39, n. 2, 2014.

WORLD HEALTH ORGANIZATION. Obesity. Preventing and managing the global epidemic. Geneva: World Health Organization; 1998. (Report of WHO Consultation on Obesity). 